\title{
Thoracoscopic fluorescence diagnosis (TFD) of pleural malignancies: experimental studies
}

\author{
R L Prosst, S Winkler, E Boehm, J Gahlen
}

Thorax 2002;57:1005-1009

See end of article for authors' affiliations

.....................

Correspondence to: Dr med J Gahlen, Chirurgische

Universitätsklinik

Mannheim,

Theodor-Kutzer-Ufer 1-3,

68167 Mannheim,

Germany;

johannes.gahlen@

chir.ma.uni-heidelberg.de

Revised version received 13 June 2002

Accepted for publication

21 July 2002

\begin{abstract}
Background: Fluorescence diagnosis (FD) using the photosensitiser 5-aminolaevulinic acid (ALA) was experimentally combined with conventional video assisted thoracic surgery (VATS) to improve tumour staging in advanced lung cancer with pleural tumour spread.

Methods: A disseminated pleural carcinosis affecting the entire pleural cavity was induced by inoculation of human adenocarcinoma cells in nude rats. After 5-7 weeks of tumour growth the animals were randomised into six groups with different photosensitisation parameters. Pleural lavage was performed either with $1.5 \%$ or $3.0 \%$ ALA solution. Photosensitisation times varied were 2,4 , or 6 hours. Conventional white light VATS was first performed to evaluate tumour growth in the pleural cavity. Fluorescence illumination of the light source, the D-light, was then used to examine the site for additional tumours which were previously invisible. The tumour fluorescence intensity was measured spectrometrically and compared with normal tissue.

Results: Compared with conventional white light VATS alone, thoracoscopic fluorescence diagnosis (TFD) detected up to $30 \%$ additional pleural malignant lesions. The highest diagnostic sensitivity was reached 6 hours after 3.0\% ALA pleural lavage. Photosensitiser accumulation in the tumour, measured indirectly by spectrometry, was up to 11 times higher than in normal tissue.

Conclusions: TFD increases sensitivity of VATS for tumour staging. It may prevent unnecessary thoracotomies in cancer patients and facilitate surgical planning.
\end{abstract}

M ost intrathoracic malignant neoplasms such as lung cancer or mesothelioma are characterised by a typically long delay between the onset of symptoms and the time of diagnosis. Currently, the best chance for long term survival lies with early diagnosis and radical resection. ${ }^{1}$ Resectability depends on the tumour stage, so precise preoperative and peroperative staging are of the utmost importance. ${ }^{2}$ However, this requires a high index of suspicion and a standardised diagnostic work up. ${ }^{1}$

The sensitivity of conventional diagnostic techniques such as computer or magnetic resonance tomography is not sufficient to ensure precise staging of intrathoracic and mediastinal lesions. Video assisted thoracic surgery (VATS) can achieve a highly accurate degree of staging by detecting tumour invasion or metastatic spread, particularly in pleural cancer. The thoracoscopic approach has been established as the procedure of choice with good results in various chest diseases such as undiagnosed pleural effusions, recurrent, post-traumatic or complicated spontaneous pneumothorax, empyema and, especially, for accurate staging of lung cancer in the resection of peripheral solitary pulmonary nodules and lung biopsy for pulmonary diffuse disease. ${ }^{3}$

Despite the good diagnostic capabilities of VATS, medical research always strives for even better results and technical conditions. In order to achieve optimum diagnostic results with VATS, approximating 100\% sensitivity by precise assessment of disseminated intrathoracic tumour spread, we combined VATS with fluorescence diagnosis (FD) - that is, thoracoscopic fluorescence diagnosis (TFD).

$\mathrm{FD}$, formerly known as photodynamic diagnosis (PDD), is increasingly used to distinguish healthy from diseased tissue in various medical disciplines. It has been used particularly in urology where it can visualise even small sized, plane tumours of the bladder. The principle of FD is based on the almost specific accumulation of administered agents (photosensitisers) in malignant cells. After intravenous, oral or topical application, the drug concentrates in tumours and remains inactive until exposed to light of a specific wavelength. When light is delivered to the cancer site, either directly or indirectly through a fibreoptic device, it causes fluorescence of the photosensitiser. Whereas earlier generations of photosensitisers such as Photofrin were already fluorescent at the time of application (exogenous photosensitisers), the photosensitiser aminolaevulinic acid (ALA) requires endogenous metabolism before it can fluoresce (endogenous photosensitisers). ALA is the natural precursor of the heme pathway. Administration of ALA, either systemically or locally, overloads the last step in the heme biosynthesis pathway of tumour cells as a result of missing negative feedback mechanisms and reduced enzymatic activities. This results in increased accumulation of protoporphyrin IX (PpIX), a metabolite of ALA. PpIX is a fluorescent agent when stimulated by light of a defined wavelength within its absorption spectrum. One main emission wavelength is within the visible light spectrum at $635 \mathrm{~nm}$ (red light). The positive red fluorescence of PpIX is even detectable in macroscopically invisible tumour foci and can indicate lesions which would be missed when illuminated with conventional white light only. ${ }^{4-8}$

\section{METHODS}

All experiments were carried out in accordance with protocols approved by national experimental animal welfare institutions.

\section{Tumour model}

Human lung cancer cells (adenocarcinoma 82/5) were cultivated on the hind leg of nude mice (Swiss cdl nu/nu) after subcutaneous injection. After reaching a tumour size of about $10 \mathrm{~mm}$ in diameter the cancer tissue was resected, sliced, and dissolved in medium RMPI-1640 (Life Technologies, UK). In parallel, 38 female nude rats (RNU rats) of mean weight $165 \mathrm{~g}$ (range 150-200) were anaesthetised with halothane (Willy Rüsch, Germany) and nitrous oxide. A 


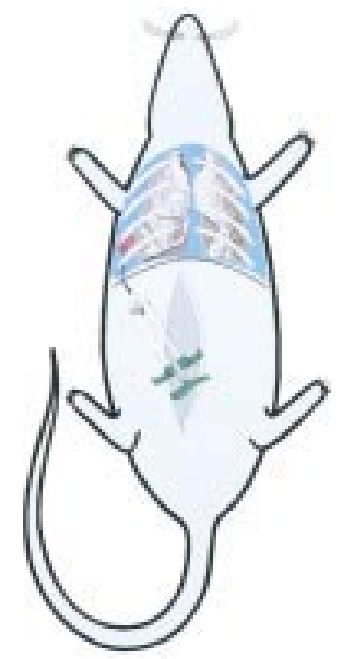

Figure 1 Tumour cell inoculation into pleural cavity of right hemithorax: laparotomy and tumour cell injection through the lateral aspect of the diaphragm under laparoscopic guidance.

laparotomy in the midline of the upper abdomen was then performed and, under visual control of a $4 \mathrm{~mm}$ laparoscope (Hopkins II, $30^{\circ}$, Karl Storz, Germany) connected to a modified CCD camera (Tricam, Karl Storz, Germany), $400 \mu \mathrm{l}$ of the tumour cell suspension was injected with a long $21 \mathrm{G}$ needle through the lateral diaphragm into the pleural cavity of the right hemithorax (fig l).

After tumour inoculation the animals were kept under standard laboratory conditions with free access to water and food in a 12 hour light/dark schedule. After 5-7 weeks of tumour growth a disseminated intrathoracic carcinosis affected the whole pleural cavity.

\section{Photosensitisation}

The animals were randomly divided into six groups with a minimum of four in each. After deep anaesthesia, local photosensitisation was achieved by pleural lavage (transdiaphragmatic access after laparotomy, see above) with 1 ml ALA solution ( $\mathrm{pH}$ 6.5, Medac, Germany) of two different concentrations (1.5\% and 3.0\%). Photosensitisation times (interval between ALA application and TFD) of 2, 4, and 6 hours were chosen. The animals were then kept under subdued light conditions to avoid photobleaching and phototoxic reactions.

\section{Thoracoscopic fluorescence diagnosis (TFD)}

Conventional white light VATS followed by TFD was performed after the photosensitisation interval using the D-light system (Karl Storz, Germany). The system is based on a $300 \mathrm{~W}$ xenon short arc lamp with special optical properties to focus high intensities of light into a light guide primarily designed for endolaparoscopic use. Two different illumination modes are possible: a conventional white light mode and a specific blue light (380-440 nm) mode for PpIX fluorescence excitation. The modes can easily be switched any time by a footswitch or directly by a button on the CCD camera head. ${ }^{5}$

The right diaphragm was incised and the laparoscope was introduced into the pleural space (fig 2). Complete examination of the entire pleural cavity was performed using white light. All tumours detected were counted and their location mapped. The same procedure followed in the blue light mode under fluorescence conditions. Previously detected tumours were evaluated according to their fluorescence. The visceral and parietal pleura was examined for additional fluorescence positive tumours which were invisible under white light.

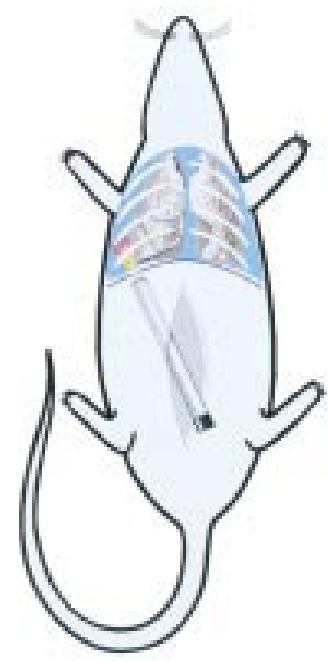

Figure 2 VATS after laparotomy and transdiaphragmatic access.

\section{Spectrometry}

The animals were killed under deep anaesthesia and the chest was opened. The fluorescence intensities of tumours and surrounding healthy tissue were measured by spectrometry. The excitation light of the D-light was transferred to the tissue via one leg of a bifurcated fibre bundle (Avantes, Germany). The common tip of the fibre was brought into contact with the lesion and the second leg of the fibre was connected to an optical multichannel analyser (OMA, Avantes, Germany). Spectral analysis was performed using specific software (SpectraWin, Avantes, Germany). Background signals were subtracted from the spectra and fluorescence intensities were measured at a typical maximal emission wavelength of PpIX $(635 \mathrm{~nm})$. From each tissue sample a number of spectral intensities were gained and averaged. The fluorescence ratio was determined by comparing the fluorescence intensity of tumours with the score for normal healthy tissue.

All tumours identified either by white light or fluorescence were removed for histological examination.

\section{RESULTS}

Injection of cancer cells into the pleural cavity led to disseminated tumour growth affecting the pleural surface of the lung, the parietal pleura of the inner chest wall, the diaphragm, and the mediastinum (fig 3). A total of 123 lesions was found (mean 3.2 tumours per animal). The maximum tumour size was about $8 \mathrm{~mm}$.

In all groups except one ( 2 hours of photosensitisation with $1.5 \%$ ALA), TFD enabled the detection of tumours which were missed with conventional VATS (fig 4). These lesions, which indicate the diagnostic value of TFD (increase in sensitivity), were mainly observed after 6 hours of photosensitisation using 3.0\% ALA solution (30\%). Photosensitisation for 4 hours gave slightly poorer results with both 3.0\% ALA (26\%) and $1.5 \%$ ALA (7\%). An even shorter time period between ALA application and TFD (2 hours) decreased the improvement in sensitivity even more (1.5\% ALA: no gain, 3.0\% ALA: $4 \%$ ). Only three of the macroscopically visible neoplasms showed no fluorescence under blue light, leading to false negative results. On the other hand, four macroscopically visible lesions which showed no fluorescence were histologically benign, leading to true negative results.

Spectrometry of fluorescence intensities, as an indirect measurement of accumulation of photosensitised tissue, showed an increasing absolute fluorescence intensity and tumour:normal tissue ratio with longer photosensitisation time in tumours of the parietal pleura (fig 5). The best fluorescence contrast between neoplasms and surrounding 

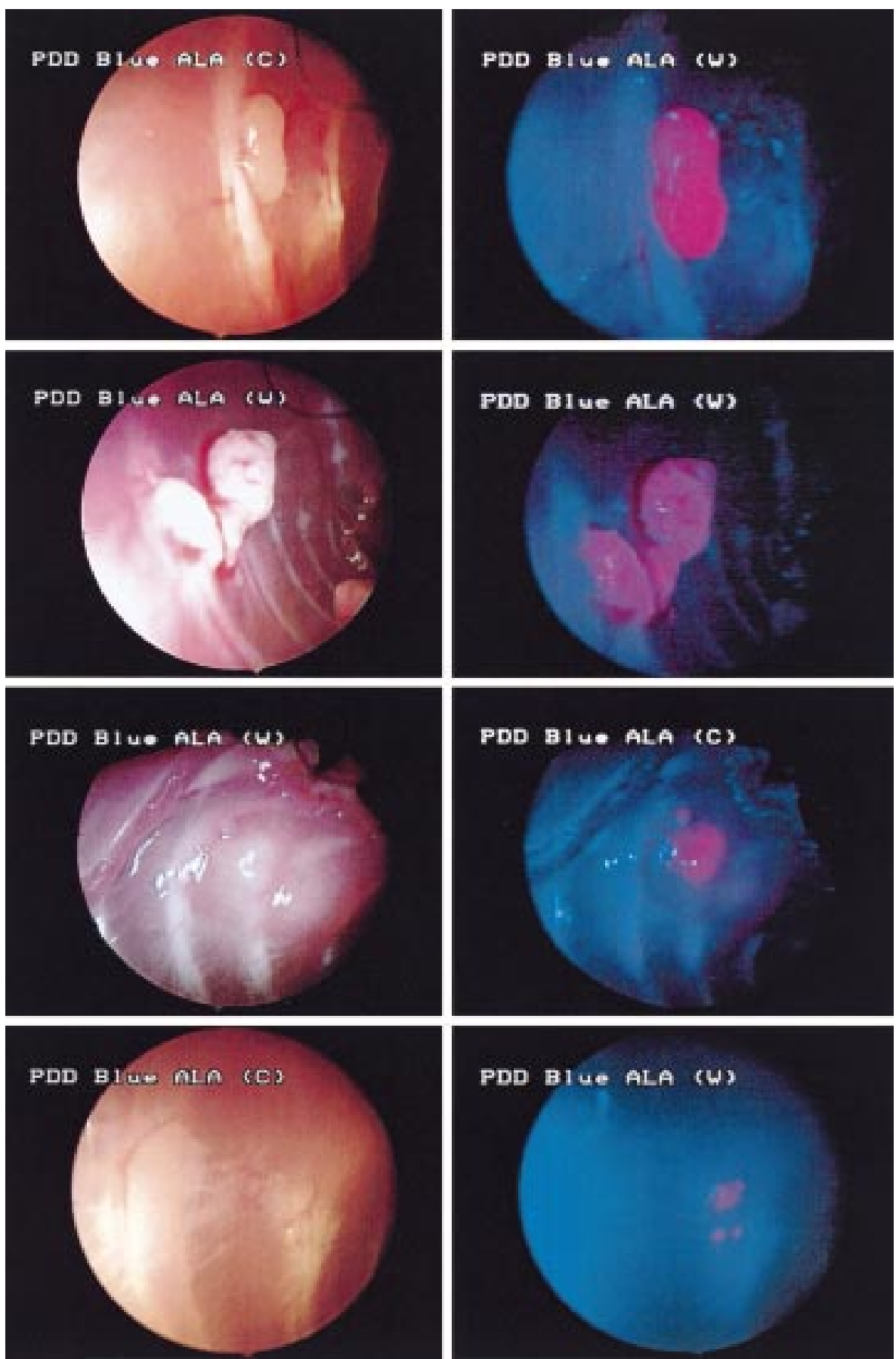

Figure 3 Thoracoscopic views under conventional white light (left) and fluorescence illumination by the D-light (right). Lesions located on the parietal pleura.

tumour free tissue $(11: 1=11$ times higher fluorescence intensity in tumour) was thus achieved after 6 hours of photosensitisation with both ALA concentrations.

A randomised number of tumours were resected after the examination and histologically confirmed as carcinomas while tissue samples thought to be healthy were tumour free.

\section{DISCUSSION}

The prognosis of intrathoracic malignancies is generally poor when the disease is disseminated. In these cases radical surgery should therefore be avoided as palliation can potentially be performed by less invasive procedures. ${ }^{9}$

The diagnosis and staging of intrathoracic malignancies requires assessment of the lung parenchyma, hilum, pleura,

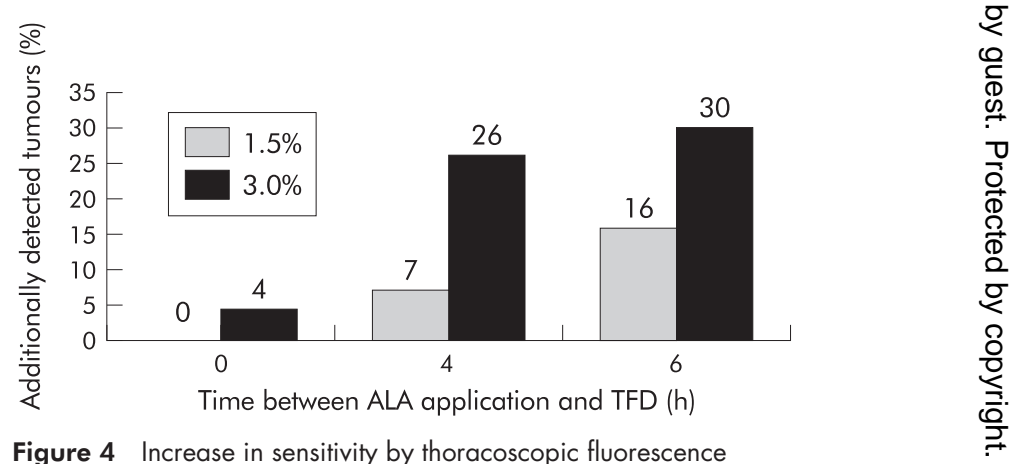

diagnosis (TFD). 


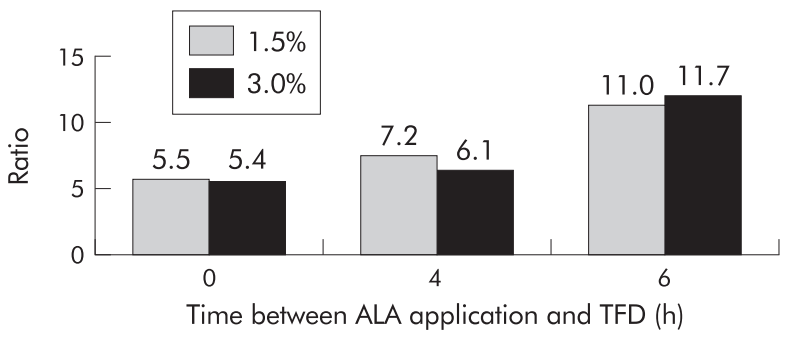

Figure 5 Fluorescence intensity ratio between tumour and normal tissue.

chest wall, and intrathoracic lymph nodes. For lung cancer this means exploration of all potential metastatic sites beyond the lung parenchyma. The pleural space may be directly or indirectly involved with the primary lung cancer.

Computed tomographic scanning is an essential diagnostic tool by which initial information regarding diagnosis and treatment can be achieved. Information on morphology, density, and vascular layout of the mass, its relationship with surrounding structures, the presence or absence of a capsule or a cleavage plane, as well as an estimate of its invasiveness can all be obtained by this first diagnostic method. However, the sensitivity and specificity are often inadequate and further information on resectability can only be achieved by invasive staging such as mediastinoscopy or VATS.

Roberts $e t$ al $^{10}$ showed that VATS was more accurate than CT scanning in the staging of 50 patients with early lung cancer (stages IA, IB, IIA, and IIB), especially the T stage. In addition, thoracoscopic examination ruled out malignancy in seven patients (14\%) with radiologically observed effusions, and identified radiologically silent malignant pleural effusions in three $(6 \%)$. Invasion of the chest wall was accurately identified by VATS in most patients. Three patients with Tl lower lobe lesions and negative mediastinoscopy were found to have involvement of the inferior mediastinal nodes (level 8 or 9) by VATS. They concluded that errors in thoracoscopic staging resulted in no inappropriate operations. However, errors in CT staging would have resulted in operations unlikely to help the patients or would have inappropriately excluded patients from surgery.

Roviaro et $\mathrm{l}^{11}$ performed VATS in 286 patients prior to curative surgical resection of lung cancer to determine resectability. Unsuspected causes of inoperability were identified in $5.7 \%$ and 11 patients who were thought to be inoperable by preoperative CT scanning were deemed to have resectable tumours. The rate of exploratory thoracotomies was reduced to less than $4 \%$. The authors recommended that VATS should be performed in every patient with lung cancer to obtain more detailed staging and to reduce the number of exploratory thoracotomies.

VATS has become the procedure of choice for patients with indeterminate peripheral lung nodules, diffuse lung disease, pleural disease and effusions, or spontaneous pneumothorax. ${ }^{12}$ It visualises the pleural cavity including the diaphragmatic visceral pleura and the lung. The procedure does not only give information on the extent of the disease itself, but allows adequate biopsy sampling. This facilitates the distinction between viable tumour and, for example, fibrotic reaction. VATS has the advantage of limiting surgical trauma and the postoperative pain associated with thoracotomy ${ }^{13}$ which results in excellent patient acceptability. It is a valuable alternative to traditional thoracotomy for various indications.

Champion et al $^{14}$ compared VATS with open thoracotomy for staging of lung cancer in 17 patients to evaluate whether video imaged thoracoscopic staging was accurate. There was complete TNM stage correlation between the closed video imaged thoracoscopic and the open thoracotomy techniques. They concluded that minimally invasive video imaged thoracoscopic staging is an accurate method for assessing the stage of lung cancer to guide rational management.

Rau et $a l^{9}{ }^{15}$ found that VATS was a sensitive technique for staging pulmonary coin lesions. Additional information was achieved in $48-49 \%$ of patients and the therapeutic strategy was changed in $34-40 \%$ as a result of distant spread of malignancy or detection of benign lesions by thoracoscopic staging. Invasive treatment such as surgical intervention was avoided in $19 \%$.

Yim ${ }^{16}$ found two unsuspected pleural metastases by VATS in 63 patients $(3.2 \%)$ clinically thought to have early lung cancer. These results were similar to those of Wain ${ }^{17}$ who also found two pleural metastases in 43 patients $(4.7 \%)$. However, in the latter study VATS missed six of 16 T2 lesions with visceral pleural invasion (38\%) and five of 11 with $\mathrm{Nl}$ disease $(45 \%)$.

Like lung cancer, mesothelioma is difficult to diagnose early. Nevertheless, disease stage is the determining factor for outcome of treatment. Poor outcome of treatment could be due to delayed diagnosis. Effective diagnostic examinations must therefore be undertaken promptly as certain forms of mesothelioma may have a better prognosis when diagnosed early and treated by multimodal therapy or intrapleural immunotherapy. ${ }^{18}$ Diagnosis depends primarily on histological examination of samples obtained by VATS. This procedure allows the best staging of the pleural cavity and can detect visceral pleural involvement, which is one of the most important prognostic factors. ${ }^{19}$ In early stage mesothelioma VATS enabled patients with stage Ia disease (involvement confined either the parietal or diaphragmatic pleura and purely inflammatory aspect in $50 \%$ of cases) to be distinguished from those with stage Ib disease (involvement of the visceral pleura).$^{20}$ In this respect, the combination of VATS with fluorescence may provide a more sensitive detection tool.

Fluorescence illumination techniques have been developed mainly for urology. Flat neoplastic lesions such as dysplasia, carcinoma in situ, or small papillary tumours of the bladder are sometimes difficult to diagnose as they grow in normal or non-specifically inflamed mucosa. The use of fluorescence endoscopy has revealed a significantly higher detection rate of these lesions than with conventional white light alone. Based on these promising clinical results, fluorescence diagnosis has also become a diagnostic tool in other medical fields. In gastroenterology, otolaryngology, and gynaecology it is used to diagnose tumours and precursors of malignant tissue, particularly flat mucosal lesions. Neurosurgeons also use fluorescence for the intraoperative detection of malignant gliomas, while in dermatology efforts have been maintained to diagnose and demarcate, for example, basal cell carcinoma. ${ }^{5}$

Our previous investigations were conducted to evaluate laparoscopic fluorescence diagnosis for gastrointestinal tumour staging. ${ }^{4-7}$ Using the same technical equipment as for the present study, we were able to show a distinctly higher rate of detection of lesions of a disseminated peritoneal carcinosis than with conventional laparoscopy alone.

The present study has shown the value of TFD in diagnosing the extent of tumour spread in the pleural cavity and in increasing the accuracy of tumour staging. TFD was technically feasible with little expenditure of time and training. Photosensitisation with 3.0\% ALA solution and 6 hours exposure time appeared to be most effective in detecting additional pleural lesions. Shorter exposure times resulted in lower sensitivity and an unacceptable number of false negative results. Furthermore, a photosensitisation time of 6 hours resulted in an excellent tumour discrimination ratio of $11: 1$.

Although theoretically available for several decades, fluorescence techniques have become more attractive for clinical use since more effective generations of photosensitisers such as ALA have been clinically implemented and tested. They were intended to reduce common side effects of photosensitisers such as skin sensitivity, nausea, vomiting and transiently raised liver transaminase levels. ALA induced PpIX photosensitisation has several advantages: (1) ALA, PpIX, and other 
intermediates are rapidly eliminated from humans, thus minimising the risk of skin phototoxicity; (2) ALA can be applied locally, providing an acceptable and convenient route of administration for patients; (3) the fluorescence ratio between tumours and surrounding healthy tissue is superior to that of other photosensitisers. ${ }^{4}$ The clinical use of TFD in humans would require either systemic (oral, intravenous) administration of ALA or pleural lavage. However, major side effects are expected to be even less after local application by pleural lavage.

In conclusion, TFD is a feasible technique for improving the preoperative diagnosis and tumour staging in patients with intrathoracic malignancies. It may prevent patients from undergoing unnecessary thoracotomies and facilitate planning of thoracotomies by increased diagnostic sensitivity.

\section{ACKNOWLEDGEMENTS}

These studies have been supported by a research grant from the University of Heidelberg (Forschungs-Foerderprogramm der Medizinischen Fakultaet Heidelberg, 178/2000).

The authors wish to thank N Decker for her invaluable help and effort and are grateful to Dr J Mattern (DKFZ Heidelberg) for support in tumour cell cultivation.

\section{Authors' affiliations}

R L Prosst, J Gahlen, Department of Surgery, University Hospital Mannheim, Ruprecht-Karls-University of Heidelberg, Germany S Winkler, E Boehm, Department of Surgery, University Hospital Heidelberg, Ruprecht-Karls-University of Heidelberg, Germany

\section{REFERENCES}

1 Ho L, Sugarbaker DJ, Skarin AT. Malignant pleural mesothelioma. Cancer Treat Res 2001;105:327-73.

2 Van Schil PE, Van den Brande F, De Maeseneer MG. Operative staging of lung cancer. Monaldi Arch Chest Dis 2000;55:299-304.
3 Petrakis I, Katsamouris A, Drossitis I, et al. Usefulness of thoracoscopic surgery in the diagnosis and management of thoracic diseases. $J$ Cardiovasc Surg (Torino) 2000;41:767-71.

4 Gahlen J, Prosst RL, Pietschmann M, et al. Laparoscopic fluorescence diagnosis (LFD) for intraabdominal fluorescence targeting of peritoneal carcinosis: experimental studies. Ann Surg 2002;235:252-60.

5 Gahlen J, Prosst RL, Herfarth Ch. Blue light illumination for minimally-invasive fluorescence detection of tumors: technology, clinical experience and future perspectives. Min Invas Ther Allied Technol experience and futur

6 Gahlen J, Prosst RL, Pietschmann M, et al. Spectrometry supports fluorescence staging laparoscopy after intraperitoneal aminolaevulinic acid lavage for gastrointestinal tumours. J Photochem Photobiol B 1999:52:131-5.

7 Gahlen J, Stern J, Laubach $\mathrm{HH}$, et al. Improving diagnostic staging laparoscopy using intraperitoneal lavage of 5-aminolevulinic acid (ALA) for laparoscopic fluorescence diagnosis. Surgery 1999:126:469-73.

8 Gahlen J, Stern J, Pressmar J, et al. Local 5-aminolevulinic acid 8 Gahlen J, Stern J, Pressmar J, et al. Local 5-aminolevulinic acid
application for laserlight induced fluorescence diagnosis of early staged colon cancer in rats. Lasers Surg Med 1999;26:302-7.

9 Rau B, Hunerbein M, Below C, et al. Video-assisted thoracic surgery. Staging and management of thoracic tumors. Surg Endosc 1998;12:133-6

10 Roberts JR, Blum MG, Arildsen R, et al. Prospective comparison of radiologic, thoracoscopic, and pathologic staging in patients with early non-small cell lung cancer. Ann Thorac Surg 1999;68: 1 154-8.

11 Roviaro GC, Varoli F, Rebuffat C, et al. Videothoracoscopic operative staging for lung cancer. Int Surg 1996;81:252-4.

12 Rieger R, Wayand W. Video-assisted thoracoscopy in diagnosis and therapy of intrathoracic diseases. Zentralb/ Chir 1997;122:1065-71.

13 Mentzer SJ, Swanson SJ, DeCamp MM, et al. Mediastinoscopy, thoracoscopy, and video-assisted thoracic surgery in the diagnosis and staging of lung cancer. Chest 1997;112(4 Suppl):239-41S.

14 Champion JK, McKernan JB. Comparison of minimally invasive thoracoscopy versus open thoracotomy for staging lung cancer. Int Surg 1996:81:235-6

15 Rau B, Hunerbein M, Barth $C$, et al. Thoracoscopic diagnosis and therapy of lung metastases. Zentralb/ Chir 1998;1 23: $1125-8$.

16 Yim AP. Routine video-assisted thoracoscopy prior to thoracotomy. Chest 1996;109: 1099-100

17 Wain JC. Video-assisted thoracoscopy and the staging of lung cancer Ann Thorac Surg 1993;56:776-8.

18 Boutin C, Monnet I, Ruffie P, et al. Malignant mesothelioma: clinical and therapeutic study. Rev Mal Respir 1999:16:1317-26.

19 Boutin C, Schlesser M, Frenay C, et al. Malignant pleural mesothelioma. Eur Respir J 1998;12:972-81.

20 Boutin C, Frenay C, Astoul P. Endoscopic diagnosis of mesothelioma. Rev Mal Respir 1999;16:1257-62. 